\title{
Die zweite Schenkkreisentscheidung des BGH als Ausgangspunkt für einen Paradigmenwechsel im Hinblick auf die Auslegung von § 817 S. 2 BGB
}

\author{
Von Priv.-Doz. Dr. Matthias Armgardt ${ }^{1}$
}

\begin{abstract}
Der nachfolgende Beitrag analysiert die zweite Schenkkreisentscheidung des $B G H,{ }^{2}$ die insbesondere die Auslegung des in $\$ 817$ S. 2 $B G B$ geregelten Kondiktionsausschlusses betrifft. Die dort aufgestellten Grundsätze entsprechen den vom Verfasser vorgeschlagenen Prinzipien zur Auslegung diese Vorschrift ${ }^{3}$ und geben Anlass zur Hoffnung auf eine grundsätzliche Korrektur der Rechtsprechung zu \$ 817 S. 2 BGB. Das Ergebnis wird im Wege einer historischen und rechtsvergleichenden Analyse untermauert. Weiterhin wird gezeigt, dass die hier vertretene Ansicht auch vom Draft Common Frame of Reference gestützt wird.
\end{abstract}

\section{Die Zweite Schenkkreisentscheidung des BGH}

Der BGH hat in seiner ersten Schenkkreisentscheidung aus dem Jahr 2005 festgestellt, dass bei sog. Schenkkreisen der bereicherungsrechtliche Rückforderungsanspruch des »Schenkers « gegen den »Beschenkten« nicht an $\$ 817$ S. 2 BGB scheitert. ${ }^{4}$ Diese Entscheidung hat überwiegend Zustimmung gefunden. ${ }^{5}$ Sie ist allerdings bei manchen Amtsgerichten auf Widerspruch gestoßen. ${ }^{6}$ Zudem hat Schmidt-Recla kürzlich vorgeschlagen, $\$ 817$ S. 2 BGB anhand ungeschriebener subjektiver Kriterien auszulegen. Im Hinblick auf die Schenkreisentscheidung sei die Nichtanwendbarkeit der Kondiktionssperre des $\S 817$ S. 2 BGB nur gerechtfertigt, wenn dadurch im Ergebnis »mathematisch oder im Leben unerfahrene Personen, die den dem System inhärenten Kollabierungseffekt nicht zu überschauen vermögen « geschützt würden. Sei sich der Leistende des Sitten- oder Gesetzesverstoßes bewusst, müsse zu seinen Lasten $\$ 817$ S. 2 BGB Anwendung finden. ${ }^{7}$

Der BGH hat diesen Ansichten in seiner zweiten Schenkkreisentscheidung vom 13.3. 2008 unter Aufrechterhaltung der ersten Schenkkreisentscheidung eine klare Absage erteilt. ${ }^{8}$ Ihm zufolge entfällt die Kondiktionssperre des $₫ 817$ S. 2 BGB nicht nur bei Ansprüche gegen die Initiatoren, sondern auch bei Ansprüchen gegen die weiteren »Beschenkten $« .{ }^{9}$ Zudem kommt nach Ansicht des BGH eine einzelfallbezogene Prüfung der Geschäftsgewandtheit und Erfahrenheit des betroffenen Gebers oder Empfängers nicht in Betracht. ${ }^{10}$ Geboten sei vielmehr eine generalisierende Betrachtungsweise. ${ }^{11}$ Innerhalb der Leistungskondiktion sei der Schutzzweck der jeweiligen nichtigkeitsbegründenden Norm maßgeblich, welcher durch den Kondiktionsausschluss nicht dadurch konterkariert werden dürfe, dass der durch sie zu verhindernde sittenwidrige Zustand perpetuiert oder weiterem sitten- und verbotswidrigem Handeln Vorschub geleistet wird. ${ }^{12}$

Nicht nur gegen die Initiatoren des Spiels, sondern auch gegen die Beschenkten der weiteren Stufen seien Bereicherungsansprüche zu gewähren, weil diese sonst ihre Schenkungen rückabwickeln könnten, während sie die ihnen geschenkten Beträge behalten könnten. Dies stelle einen Wertungswiderspruch dar. ${ }^{13}$ Im Ergebnis sorgt die neueste BGH-Rechtsprechung also dafür, dass das gesamte »Spiel« rückabgewickelt wird, wenn die einzelnen Spieler darauf bestehen.

\section{Die Zweite Schenkkreisentscheidung als sinnvolle Korrektur der Schwarzarbeits- und Gewinnaus- schüttungsentscheidung}

Die neueste Entscheidung des BGH zur Schenkkreisthematik ist uneingeschränkt zu begrüßen. Sie stellt eine sinnvolle Korrektur der nicht immer glücklichen BGH-Rechtsprechung zu $\$ 817$ S. 2 BGB dar. ${ }^{14}$ Der BGH folgt der Sache nach dem von uns vorgetragenen Vorschlag, $\$ 817$ S. 2 BGB einerseits insoweit teleologisch zu reduzieren, als der Normzweck des Verbotsgesetzes und generalpräventive Erwägungen vorrangig zu berücksichtigen sind, andererseits aber auf eine darüber hinausgehende Abwägung der Interessen der Parteien im Einzelfall zu verzichten. ${ }^{15}$

In der umstrittenen Schwarzarbeitsentscheidung ${ }^{16}$ hatte der BGH gerade anders entschieden: Der BGH lehnte die Anwendung des $\$ 817$ S. 2 BGB ab, obwohl die vom Gesetzgeber erwünschte generalpräventive Wirkung seines Erachtens bereits durch die Gefahr einer Strafverfolgung und der Nachzahlung von Steuern und Sozialabgaben hinreichend stark ausgeprägt war. Das Argument gegen die Anwendbarkeit des $\$ 817$ S. 2 BGB hattealsokeinen generalpräventiven Charakter. Vielmehr nahm der BGH eine Abwägung der Parteiinteressen vor. Da der Auftraggeber meist wirtschaftlich stärker sei, müsse er keinesfalls besser behandelt werden als der wirtschaftlich schwächereSchwarzarbeiter. ${ }^{17}$ Daher gewährte der BGH dem Schwarzarbeiter im Ergebnis einen bereicherungsrechtlichen Ausgleichsanspruch auf Wertersatz.

Der überwiegende Teil des Schrifttums hat die Schwarzarbeitsentscheidung zu Recht entschieden abgelehnt. ${ }^{18}$ S. Lorenz spricht

1 Der Autor ist Privatdozent (Universität zu Köln) und derzeit Lehrstuhlvertreter an der LMU München.

2 BGH NJW 2008, $1942 \mathrm{f}$.

3 ArMgardT NJW 2006, 2070, 2073.

4 BGH NJW 2006, S. 45, 46. Anders noch das OLG Köln, das bereicherungsrechtliche Rückabwicklungsansprüche an $\$ 817$ S. 2 BGB scheitern ließ, NJW 2005, S. 3290, $3291 \mathrm{f}$.

5 Armgardt NJW 2006, 2070, 2073; Staudinger/S. Lorenz (2007) $\$ 817$ Rdn. 10; Mölle R NJW 2006, S. 268, 270; K. S C H м i d TuS 2006, 265.

6 Z.B. AG Siegburg, NJW-RR 2007, 1431.

7 Schmidt-Recla JZ 2008, 60, 67.

8 BGH III ZR 282/07 = NJW 2008, $1942 \mathrm{f}$.

9 Ebenso OLG Köln, NJW 2006, 3288.

10 Ausdrücklich gegen Schmidt-Recla BGH NJW 2008, 1942.

11 Gegen eine darüber hinausgehende Abwägung der Parteiinteressen schon ausdrücklich A r M G A R D T NJW 2006, 2070, 2073. Ähnlich Staudinger/S. LORENZ (2007) \$817 Rdn. 10.

12 BGH NJW 2008, 1942. Ebenso Armgardt NJW 2006, 2073 und Staudinger/S. Lor EN Z (2007) \$817 Rdn. 10.

13 BGH NJW 2008, 1942 mit Hinweis auf OLG Köln, NJW 2006, 3288.

14 Ein Überblick findet sich bei Arm G A R T NJW 2006, $2070 \mathrm{ff}$.

15 Armgard T NJW 2006, 2070, 2073.

16 BGHZ 111, 308 = NJW 1990, $2542 \mathrm{f}$.

17 BGHZ 111, 308, 313 (= NJW 1990, $2542 \mathrm{f}$ ).

18 Ablehnend vor allem MünchKomm/L i в в 4. Aufl. (2004),\$817 Rdn. 37; Staudinger/S. Lorenz (2007) \$817 Rdn. 10; Ti e D T K E DB 1990, 2307, 2310; Larenz/Canaris, Lehrbuch des Schuldrechts, Zweiter Band, 
insofern treffend von einer »über die Maßen strapazierten Restriktion des $\$ 817$ S. $2 \ldots$ contra legem«. Das Argument, der Schwarzarbeiter sei der wirtschaftlich schwächere Teil und seine Vorleistung bringe dem Auftraggeber einen unentgeltlichen Vorteil, reiche zur Ausschaltung der Kondiktionssperre nicht aus. ${ }^{19}$ Die Auffassung von Lorenz verdient volle Zustimmung. Sie deckt sich im Wesentlichen mit unserem Vorschlag, $₫ 817$ S. 2 BGB zwar unter den Vorbehalt des Normzweckes und der Generalprävention zu stellen, ${ }^{20}$ aber keinesfalls eine darüber hinausgehende Abwägung der Parteiinteressen vorzunehmen. Insofern ist schlicht die gesetzgeberische Entscheidung zu respektieren. Ein Gericht, dass sich darüber hinwegsetzt, entscheidet in der Tat contra legem und missachtet seine Gesetzesbindung. Ein derart drastisches Vorgehen wäre allenfalls dann zu rechtfertigen, wenn bei Anwendung der gesetzlichen Normen unauflösbare und unerträgliche Wertungswidersprüche eintreten würden. Davon kann in Zusammenhang mit der Schwarzarbeitsthematik aber keine Rede sein. Daher ist die Schwarzarbeitsentscheidung schon aus methodischen Gründen abzulehnen.

Auch bei der Gewinnausschüttungsentscheidung, ${ }^{21}$ bei der es um bereicherungsrechtliche Rückforderungsansprüche in Bezug auf geleistete Gewinnausschüttungen zur Erfüllung eines wegen Gewinnsucht sittenwidrigen Anlagevertrages ging, argumentierte der BGH im Hinblick auf die Frage, ob die Kondiktionssperre des $₫ 817$ S. 2 BGB anwendbar sei, nicht mit Normzweck oder Generalprävention, sondern gestattete sich eine freie Abwägung der konkreten Parteiinteressen. Allerdings bejahte er in diesem Fall die Anwendbarkeit des $\$ 817$ S. 2 BGB. Das vom BGH gefundene Ergebnis ist korrekt, denn der Gesetzgeber schreibt die Anwendung von $\$ 817$ S. 2 BGB vor; die Begründung des $\mathrm{BGH}$ ist hingegen genauso wenig haltbar, wie Ergebnis und Begründung der Schwarzarbeitsentscheidung.

Die zweite Schenkkreisentscheidung ist der Schwarzarbeitsentscheidung und der Gewinnausschüttungsentscheidung insofern diametral entgegengesetzt, als der BGH eine über den Schutzzweck der Norm bzw. über den Gedanken der Generalprävention hinausgehende Abwägung der Parteiinteressen bezogen auf den konkreten Einzelfall gerade ablehnt. Insofern darf man hoffen, dass die zweite Schenkkreisentscheidung eine über die Entscheidung des Falles hinausgehende Kurskorrektur zur Frage der Auslegung des $\$ 817$ S. 2 BGB enthält und dass der BGH den neu eingeschlagenen Weg in Zukunft auch beibehalten wird.

\section{Zu den Kriterien der teleologischen Reduktion des § 817 S. 2 BGB}

Die zweite Schenkkreisentscheidung des BGH stellt klar und zutreffend heraus, dass eine »einzelfallbezogene Prüfung der Geschäftsgewandtheit und Erfahrenheit des betroffenen des Gebers oder Empfängers" nicht vorzunehmen sei. Vielmehr sei eine "generalisierende Betrachtungsweise « geboten. ${ }^{22}$ Dabei versteht der BGH in diesem Kontext unter "generalisierender Betrachtungsweise" den "Schutzzweck der jeweiligen nichtigkeitsbegründenden Norm«, der nicht konterkariert werden dürfe. Auch in diesem Punkt ist dem BGH zuzustimmen. ${ }^{23}$ Die Gedanken des Normzwecks und der Generalprävention, die sich gegenseitig ergänzen, ${ }^{24}$ was auch die Begründung der zweiten Schenkkreisentscheidung zeigt, ${ }^{25}$ sind die einzigen Kriterien, von denen man die Anwendbarkeit des $\$ 817$ S. 2 BGB abhängig machen darf. Es ist hingegen kein Raum für eine Abwägung der Parteiinteressen - auch nicht in generalisierender oder typisierender Form. Dagegen hatte der BGH in der Schwarzarbeitsentscheidung eine typisierende und in der Gewinnausschüttungsentscheidung eine auf den konkreten Einzelfall bezogene Abwägung der Parteiinteressen vorgenommen. ${ }^{26}$ Die zweite Schenkkreisentscheidung stellt zumindest klar, dass für eine auf den konkreten Einzelfall bezogene Abwägung der Parteiinteressen kein
Raum ist. Es ist zu hoffen, dass der BGH unter der "gebotenen generalisierenden Betrachtungsweise « auch in Zukunft nur Normzweck und Generalprävention verstehen wird - und nicht etwa für den Fall, dass diese Kriterien nicht zum Zuge kommen, doch noch auf eine typisierte Abwägung der Parteiinteressen rekurrieren wird. Denn das hieße, den methodischen Fehler, der der Schwarzarbeits- und Gewinnausschüttungsentscheidung zugrunde liegt, zu wiederholen.

\section{Zu den historischen Hintergründen und zur ratio der Regelung des § 817 S. 2 BGB}

Um die hier vertretene Auffassung zu stützen, sollen nachfolgend einige Argumente zur Rationalität des $₫ 817$ S. 2 BGB vorgetragen werden, wobei vorausgesetzt wird, dass die Norm im hier vertretenen Sinne, also unter dem Vorbehalt des Normzwecks und der Generalprävention, ausgelegt wird. Eine darüber hinausgehendeeinzelfallbezogene oder typisierende - Abwägung der Parteiinteressen, wie sie in der Schwarzarbeits- und der Gewinnausschüttungsentscheidung des BGH anzutreffen ist, beruht letztlich darauf, dass die Rechtsprechung $\$ 817$ S. 2 BGB für eine völlig missglückte und daher auch aufEbene der Betrachtung der Parteiinteressen zu korrigierende Norm hält. Im Folgenden soll gezeigt werden, dass diese Auffassung nicht gerechtfertigt ist.

Die Vorschrift des $₫ 817$ S. 2 basiert auf römischem Recht. ${ }^{27}$ Der ihr zugrunde liegende Gedanke besteht darin, für den Fall, dass beide Parteien außerhalb der Rechtsordnung stehen, denjenigen zu bevorzugen, der den herausverlangten Vorteil besitzt, also den in Anspruch genommenen Bereicherungsschuldner: in pari turpitudine melior est causa possidentis. ${ }^{28}$ Der hinter $\$ 817$ S. 2 BGB stehende Gedanke ist schlicht folgender: Wer die Rechtsordnung um Schutz seiner Interessen ersucht, darf nicht selbst außerhalb der Rechtsordnung stehen. ${ }^{29}$ Dieses Prinzip ist in höchstem Maße vernünftig. Die Rechtsordnung darf sich nicht zum Handlanger von nicht rechtstreuen Anspruchstellern machen lassen. Wer sich außerhalb der Rechtsordnung bewegt, darf diese auch dann nicht zur Durchsetzung seiner Interessen in Anspruch nehmen, wenn eine Parteiabwägung im Einzelfall oder auf typisierendem Niveau

Besonderer Teil, 2. Halbband, 13. Aufl., 1994, \$ 68 III 3 g, 167. Kritisch auch WEY ER WM 2002, 627, 632. Zustimmend aber KöH LER JZ 1990, 466, 469 und Erman/H.P.We S T E R M A N N 11. Aufl. 2004, \$817 Rdn. 15. 19 Staudinger/S. Lorenz (2007) \$817 Rdn. 10.

20 So auch bereits CA N A R I S in: Festschrift für Ernst Steindorff, 1990, S. 519, $523 \mathrm{ff}$. Zustimmend WENDEHORST in: Bamberger/Roth BGB, 2003, $\$ 817$ Rdn. 20.

21 BGH, NJW-RR 1993, 1457 ff. Dazu A r M g a R D t NJW 2006, 2070, 2071 und 2073.

22 BGH NJW 2008, 1942

23 So schon Armgard T NJW 2006, 2073.

24 So schon Armgard T NJW 2006, 2073.

25 BGH NJW 2008, 1942: »Innerhalb der Leistungskondiktion ist vielmehr maßgebend der Schutzzweck der jeweiligen nichtigkeitsbegründenden Norm, welcher durch den Kondiktionsausschluss nicht dadurch konterkariert werden darf, dass der durch sie zu verhindernde sittenwidrige Zustand perpetuiert oder weiterem sitten- und verbotswidrigem Handeln Vorschub geleistet würde ....

26 S. o. unter II.

27 Siehe vor allem Paulus D. 12,5,8: ». . porro autem si et dantis et accipientis turpis causa sit, possessorem potiorem esse et ideo repetitionem cessare ...". Dazu ausführlich HoNSELL Die Rückabwicklung sittenwidriger oder verbotener Geschäfte, 1974, S. 98 ff. dessen Vorschlag, \$817 S. 2 auf die Fälle der Deliktsanstiftung und der causa futura zu beschränken, sich nicht durchgesetzt hat. Siehe Honsell a. a. O., S. $136 \mathrm{ff}$. und $147 \mathrm{ff}$.

28 Dieser Gedanke ist, wie Honsell (Fn.27), S. 88 Fn. 1 gezeigt hat, viermal überliefert: Ulpian D. 3,6,5,1; Paulus D. 12,5,8; Papinian D. 12,7,5 pr.; C. $4,7,2$.

29 Dies wird seit langem als Lehre von der Rechtsschutzverweigerung bezeichnet. Siehe nur Honsell (Fn. 27), S. 60 ff. Dieser Gedanke findet sich bei Me D I C U s Bürgerliches Recht, 19. Aufl., 2002, Rdn. 697; MünchKomm/Li в в $\$ 817$ Rdn. 9; RE U T E R/M A R T I E K Ungerechtfertigte Bereicherung, 1983, S. $204 \mathrm{f}$. 
den Anspruchssteller als schutzwürdiger erscheinen lässt als den Anspruchsgegner. Es ist kein Raum für einen subtilen Interessenausgleich zwischen zwei rechtsuntreuen Bürgern. Dass der Anspruchsgegner davon profitiert, ist ein rein faktischer Reflex dieser Betrachtung. Er ist eben beatus possidens, ein glücklich Besitzender - glücklich, weil die Rechtsordnung seinen ebenfalls rechtsuntreuen Gegner nicht schützen darf. Treffend hat Honsell darauf hingewiesen, dass das eigentlich dem Besitzrecht entstammende Prinzip »in pari turpitudine melior est causa possidentis« schlicht den Gedanken ausdrückt, dass bei Verweigerung des Rechtsschutzes wegen beiderseitigen Gesetzes- bzw. Sittenverstoßes die faktische Innehabung zum Sieg im Prozess führt und dass dies kein spezifisch juristisches Problem, sondern ein »Gebot der Logik« ist. ${ }^{30}$ Dieses im römischen Recht anzutreffende Prinzip hat auch nach rund zwei Jahrtausenden nichts an Rationalität eingebüßt. Es ist, wie später genauer gezeigt werden wird, in den meisten europäischen Rechtsordnungen bekannt und wird bei der Erörterung der Problemlösungen beachtet.

Anders ist freilich der Fall zu beurteilen, dass Normzweck und Generalprävention ein anderes Ergebnis erzwingen; denn hier macht sich die Rechtsordnung nicht zum Handlanger eines rechtsuntreuen Bürgers, sondern verfolgt autonome Ziele, was an dieser Stelle des Zivilrechts ausnahmsweise möglich ist, weil sowohl Anspruchssteller als auch Anspruchsgegner außerhalb der Rechtsordnung stehen und keiner von beiden schutzwürdig ist. Honsell meint allerdings, dass »volkspädagogische Zecksetzungen dieser Art im Zivilrecht keinen Ort haben $\ll .{ }^{31}$ Es ist sicherlich richtig, dass öffentliche Interessen bei der im Zivilrecht gebotenen Abwägung der Parteiinteressen grundsätzlich keine Rolle spielen sollten. Allerdings liegt hier eine absoluter Ausnahmefall vor: beide Parteien bewegen sich außerhalb der Rechtsordnung, so dass weder die Interessen der einen, noch der anderen Partei schutzwürdig sind und sich aus ihnen daher kein Entscheidungsprinzip ableiten lässt. Denn der Anspruchsgegner behält die Sache bei Anwendung des $\$ 817$ S. 2 BGB nicht deshalb, weil er schutzwürdig ist, sondern nur zufällig als Reflex des Gedankens, dass der Anspruchsgegner die Rechtsordnung zur Durchsetzung seiner Interessen aufgrund seiner mangelnden Rechtstreue nicht in Anspruch nehmen darf. Mangels anderer Wertungskriterien ist in diesem Fall daher die Verfolgung autonomer öffentlicher Interessen möglich und vorrangig geboten. ${ }^{32}$ Es sei aber ausdrücklich zugestanden, dass es sich hier um eine Singularität handelt. Keinesfalls soll einem allgemeinen Vorrang öffentlicher Interessen vor den Interessen der Parteien auf dem Gebiet des Privatrechts das Wort geredet werden.

\section{Kombination der vorhandenen Lehrmeinungen als dogmatische Basis}

Die hier vertretene Auffassung lässt sich nicht eindeutig einer der kürzlichnochvonSchmidt-Reclaim ÜberblickpräsentiertenLehrmeinungen zuordnen. ${ }^{33}$ Es zeigt sich vielmehr, dass diese jeweils einzelne richtige Aspekte betonen, sich aber nicht gegenseitig ausschließen und daher auch nicht gegeneinander ausgespielt werden dürfen. Soweit Canaris der Straftheorie zugesteht, in ihr stecke ein wahrer Kern, nämlich der Gedanke der Generalprävention, ${ }^{34}$ ist ihm zu folgen: die Beachtung der Generalprävention ist ein sinnvolles und legitimes Kriterium zur Auslegung und Begrenzung des $\$ 817$ S. 2 BGB. ${ }^{35}$ Der Gedanke der Generalprävention ist auch nicht vom Normzweck ${ }^{36}$ zu trennen. ${ }^{37}$ Denn der Normzweck wird in der Regel generalpräventive Erwägungen umfassen, wenngleich er nicht in allen Fällen darauf zu reduzieren sein wird. Daher ergänzen sich die beiden Ansätze, ohne einander auszuschließen..$^{38}$ Außerdem benötigt man zur Ableitung der hier vertretenen Ansicht auch die Lehre von der Rechtschutzverweigerung; denn der von ihr formulierte Grundgedanke, dass die Interessen der streitenden Parteien und insbesondere des Bereicherungsgläubigers nicht ins Gewicht fallen, weil beide außerhalb der Rechtsordnung stünden und daher nicht schutzwürdig seien, ist zu Rechtfertigung der vorrangigen Berücksichtigung der Generalprävention und des Normzweckes notwendig. Andernfalls wäre insbesondere für derartige Erwägungen kein Raum, weil das Zivilrecht grundsätzlich nurdiewiderstreitenden Parteiinteressen berücksichtigen darf und nicht darüber hinausgehende öffentliche Belange.

Die hier vertretene Auffassung lässt sich also nur unter Berücksichtigung der tragenden Gründe sämtlicher vertretener Lehren dogmatisch begründen. ${ }^{39}$ Es wäre daher verfehlt, eine einzige Lehrmeinung unter Verwerfung der übrigen als allein richtig ausmachen zu wollen. Eine Kombination der Ansichten führt auch keineswegs zu Widersprüchen. Die Theorien verhalten sich zueinander vielmehr komplementär.

\section{Rechtsvergleichende Betrachtung}

Im Folgenden soll anhand einer rechtsvergleichenden Betrachtung festgestellt werden, ob in anderen europäischen Rechtsordnungen überzeugendere Lösungen als die hier vorgeschlagene auffindbar sind..$^{40}$ Dabei werden die Rechtsordnungen der ehemals sozialistischen Länder, die vor allem eine Konfiskationslösung kennen, wegen des völlig anderen Grundprinzips ausgeklammert. ${ }^{41}$ Die meisten europäischen Rechtsordnungen weisen einen Bezug zum römisch-rechtlichen Prinzip »in pari turpitudine melior est causa possidentis« auf. Bei dem Vergleich muss berücksichtigt werden, dass das Abstraktionsprinzip nur im deutschen Recht anzutreffen ist, während in Österreich und der Schweiz das Kausalprinzip, in Frankreich, Italien und den meisten anderen Rechtsordnungen hingegen das Konsensualprinzip gelten, was grundlegende Bedeutung für die Funktion des Bereicherungsrechts in den einzelnen Rechtsordnungen hat.

\section{Rechtsordnungen mit Normen, die auf einen beider- seitigen Gesetzes- oder Sittenverstoß abstellen}

\section{a) Spanien}

Der spanische Código Civil enthält eine Regelung, die wie $\$ 817$ S. 2 BGB auf einen beiderseitigen Rechtsverstoß abstellt. Art. 1306 Ziff. $1 \mathrm{CC}^{42}$ regelt die Rechtsfolgen für den Fall, dass beiden

30 Honsell (Fn. 27), S. 89.

31 Anders Honsell (Fn. 27), S. 59. Ähnlich Bü R Ge Rechtsdogmatik und Wirtschaft, 1987, S. 90.

32 Noch weiter geht CA N A R I s in: Festschrift f. Steindorff, 1990, S. 519, 524 (v. a. Fn. 24) mit Hinweis auf den generalpräventiven Charakter des Schadensersatzrechts.

33 SCHMidt-RECLA JZ 2008, S. 62 ff.

34 Canaris, (Fn. 20), S. 519, $523 \mathrm{f}$.

35 So schon A r m G A R D T NJW 2006, S. 2073. Keinesfalls war und ist es mein Anliegen - wie Schmid t-Recla meint (JZ 2008, S.63) - die Straftheorie wieder zu beleben. Es geht schlicht um die Anerkennung des Gedankens der Generalprävention als sinnvolles Kriterium für die Auslegung des $\$ 817$ S. 2 .

36 Diese Lehre geht auf v. Ca e m merer zurück, SJZ 1950, Sp. 646, 649 f. Dazu Sch mid t-Recla JZ 2008, S. 62.

37 So auch die Begründung des BGH in der zweiten Schenkkreisentscheidung, NJW 2008, 1942, die beide Aspekte erwähnt.

38 So schon Armgard T NJW 2006, S. 2073.

39 Anders SснміDт-RECLA JZ 2008, S. $62 \mathrm{ff}$.

40 Dazu eingehend SCHLECH TIEM Restitution und Bereicherungsausgleich in Europa, Bd. 1, 2000, S. 216 ff. und 637 ff.

41 Dazu, insbesondere zum polnischen Recht SCHMidt-Re C L A JZ 2008, S. 66 .

42 Art. 1306: Si el hecho en que consiste la causa torpe no constituyere delito ni falta, se observarán las reglas siguientes: 1 . Cuando la culpa esté de parte de ambos contratantes, ninguno de ellos podrá repetir lo que hubiera dado a virtud del contrato, ni reclamar el cumplimiento de lo que el otro hubiese ofrecido. 2. Cuando esté de parte de un solo contratante, no podrá éste repetir lo que hubiese dado a virtud del contrato, ni pedir el cumplimiento de lo que se le hubiera ofrecido. El otro, que fuera extraño a 
Parteien (ambos contratantes) culpa im Hinblick auf eine causa torpe vorzuwerfen ist und ordnet eine Rückabwicklungssperre an. ${ }^{43}$ Dabei ist unter causa torpe ein einfacher Gesetzes- oder Sittenverstoß zu verstehen im Gegensatz zum qualifizierten Gesetzesverstoß, der auf eine Strafnorm Bezug nimmt. ${ }^{44}$ In Rechtsprechung und Literatur findet sich der Hinweis auf die römischrechtlichen Prinzipien »nemo auditur propriam turpitudinem allegans" und das bereits erwähnte "in pari turpitudine melior est causa possidentis «. ${ }^{45}$ In der Literatur werden hinsichtlich der ratio der Norm wie in Deutschland Generalprävention, Straffunktion, Schutzzweck der Norm und Rechtsschutzverweigerung hier begründet mit der Würde der Gerichte - erörtert. ${ }^{46}$ Auch der von uns vertretene Gedanke, dass jedes dieser Prinzipien seine Berechtigung hat, findet sich in der spanischen Literatur. ${ }^{47}$ Der Blick ins spanische Recht zeigt, dass dort mit denselben Argumenten wie im deutschen Recht gearbeitet wird. Die Rechtsprechung trifft weitgehend schwer prognostizierbare freie Ermessensentscheidungen. ${ }^{48}$ Die spanische Rechtsprechung ringt offensichtlich mit ähnlichen Problemen wie die deutsche. Eine systematische Lösung, die mit den hier vorgeschlagenen Prinzipien konkurrieren könnte, hat sich bislang noch nicht herauskristallisiert.

\section{b) Italien}

Auch das italienische Recht kennt mit Art. 2035 Codice Civile ${ }^{49}$ eine an $\$ 817$ S. 2 BGB erinnernde Vorschrift, die die Rückforderung einer Leistung ausschließt, wenn sowohl Empfänger als auch Leistender (anche da parte sua) gegen die guten Sitten verstoßen haben. Über Sinn und Zweck der Vorschrift besteht die allseits anzutreffende Uneinigkeit: das Verbot der Vorteilsziehung aus dem eigenen sittenwidrigen Verhalten, der Gedanke der Privatstrafe und das Prinzip der Eigenverantwortlichkeit werden ins Feld geführt. ${ }^{50}$ Auffällig ist allerdings, dass im italienischen Recht, dem das Konsensualprinzip zugrunde liegt, Art. 2035 CC nicht die Vindikation sperrt. ${ }^{51}$ Dadurch wird der Anwendungsbereich der Norm erheblich eingeschränkt, denn der Vindikation kommt in Rechtsordnungen, die das Konsensualprinzip anwenden, wesentlich häufiger zur Anwendung, als im auf dem Abstraktionsprinzip basierenden deutschen Recht. ${ }^{52}$ Weiterhin ist erwähnenswert, dass der Fall des Gesetzesverstoßes nicht ausdrücklich in Art. 2035 CC geregelt ist. ${ }^{53}$ Rechtsprechung und Literatur greifen auf das römisch-rechtliche Prinzip »nemo auditur suam turpitudinem allegans « zurück, ${ }^{54}$ die Lösungen werden unter Differenzierung zwischen grundsätzlich nicht rückabzuwickelnden »unmoralischen « und »bloß unerlaubten« Verträgen gesucht, sind einzelfallbezogen und schwer prognostizierbar. ${ }^{55}$ Daher kann man aus dem italienischen Recht für unsere Problemstellung wenig gewinnen.

\section{Rechtsordnungen mit Normen, die auf einen ein- seitigen Gesetzes- oder Sittenverstoß abstellen}

\section{a) Schweiz}

Art. 66 des schweizerischen Obligationenrechts normiert eine Rückforderungssperre für den Fall, das jemand »in der Absicht, einen rechtswidrigen oder unsittlichen Erfolg herbeizuführen" geleistet hat. Abweichend von $\$ 817$ S. 2 BGB regelt Art. 66 OR nicht den Fall eines beiderseitigen, sondern eines einseitigen Rechts- oder Sittenverstoßes. Bislang hat das schweizerische Bundesgericht diese Norm sehr weit ausgelegt und auf die Rückforderung aller Leistungen, die auf Grund eines rechts- oder sittenwidrigen Vertrages erbracht wurden, bezogen. ${ }^{56}$ Dies wurde in der Literatur seit Jahrzehnten heftig kritisiert, ${ }^{57}$ was kürzlich zu einer grundsätzlichen Änderung der Rechtsprechung des Bundesgericht geführt hat: in seiner Entscheidung vom 12.6.2008 stellte es klar, dass »die Rückforderung gemäß Art. 66 OR nur ausgeschlossen ist, wenn die Leistungen zur Anstiftung oder Beloh- nung eines rechts- oder sittenwidrigen Verhaltens erfolgten (Gaunerlohn). Sofern der Zweck der verletzten Norm nicht eindeutig den Ausschluss der Rückerstattung bereits erbrachter Leistungen erfordert (...) « seien »diese daher im Fall der Vertragsnichtigkeit zurückzuerstatten. ${ }^{58}$ Damit ist ein jahrzehntelanges Schisma zwischen Rechtsprechung und Literatur überwunden. Aus unserer Sicht entscheidend ist die Tatsache, dass der Schutzzweck der Norm, und damit vor allem generalpräventive Erwägungen, vorrangig zu prüfen sind. Insofern findet die von uns vorgeschlagene Lösung des Vorbehalts von Normzweck und Generalprävention durch die neueste schweizerische Rechtsprechung Unterstützung.

\section{Rechtsordnungen ohne gesetzliche Regelungen zum Problem der beiderseitigen Sittenwidrigkeit bzw. des beiderseitigen Gesetzesverstoßes}

\section{a) Österreich}

Das österreichische Recht kennt keine normierte Kondiktionssperre, die dem $\$ 817$ S. 2 BGB vergleichbar wäre. Bei einem wegen Gesetzes- oder Sittenverstoßes gemäß $\$ 879$ ABGB unwirksamen Vertrag entscheidet nach Rechtsprechung und Literatur der Normzweck über die Rückforderungsmöglichkeit. ${ }^{59}$ Eine Kondiktionssperre wird im Einzelfall durch Annahme des Entstehens einer Naturalobligation konstruiert. ${ }^{60}$ Der OGH hatte im Jahre 1997 einen den beiden Schenkkreisentscheidungen ähnlichen Fall zu entscheiden (»Pyramidenspiel«), die Rückabwicklung entgegen $\$ \$ 1174$ I 1; 1432 ABGB bejaht und dabei zur Begründung den Zweck der Verbotsnorm herangezogen. ${ }^{61} \mathrm{Im}$ Ergebnis ist daher bezüglich dieser Fallgruppe kein Unterschied zwischen der Rechtsprechung des BGH und des OGH zu verzeichnen. Abgesehen von dem Hinweis auf den Normzweck lässt sich das österreichische Recht für die hier zu behandelnde Problematik daher nicht fruchtbar machen.

la causa torpe, podrá reclamar lo que hubiera dado, sin obligación de cumplir lo que hubiera ofrecido.

43 Art. 1306 Ziff. 2 regelt hingegen die Rechtsfolgen für den Fall, dass nur eine Partei schuldhaft einen Gesetzes- oder Sittenverstoß begangen hat: dann ist nur für diese Partei die Rückforderung ausgeschlossen.

44 Dazu Art. 1305 CC.

45 SChlechtriem (Fn. 40) S. 223 Rdn. 240.

46 López Beltrán, S. 329 ff. und Albaladejo/Delgado EcheVER Rí A. 2. Aufl., Art. 1305, 444 f.

47 Dí ez-Picazo, Fundamentos I, 5. Aufl., 476 f, der allerdings daraus den Schluss zieht, man komme an Einzelfalllösungen nicht vorbei.

48 SChleChtriem (Fn. 40) S. 656 Rdn. 500.

49 Art. 2035 CC: „Chi ha eseguito una prestazione per uno scopo che, anche da parte sua, costituisca offesa al buon costume non puó ripetere quanto ha pagato«.

50 SChlechtriem (Fn. 40), S. 651 Rdn. 488 m. w. N.

51 Gallo Tr. Sacco, Arricchimento senza causa, 189, m. w. N.

52 Treffend Schlechtriem (Fn. 40) S. 653 Rdn. 494 ff.

53 Zur Tendenz, die Regelung dennoch auf diesen Fall anzuwenden G A L L O (Fn. 51), S. 186.

54 Schlechtriem (Fn. 40), S. 221 Rdn. 238.

55 SChlechtriem (Fn. 40), S. 221 Rdn. 238.

56 Siehe vor allem BGE 102 II 401 E. 4 S. 409.

57 Die Kritik geht auf v. T u r R zurück. Dazu Hon S L L (Fn. 27), S. 118 ff. Aus neuerer Zeit Pe t i t p I e R R e Commentaire Romand, N. 4 zu Art. 66 OR; S C H U L I N Basler Kommentar, N. 4 fzu Art. 66 OR; B U C H E R Schweizerisches Obligationenrecht, Allgemeiner Teil, 2. Aufl., Zürich 1988 S. 678 ff.; GAU C H /S C H LU E P/S C H M I D/RE Y Schweizerisches Obligationenrecht, Allgemeiner Teil, Bd. I, 8. Aufl., 2003, Rdn. 1548 ff.; S C H W E N Z E R Schweizerisches Obligationenrecht, Allgemeiner Teil, 4. Aufl., 2006 S. 393 f; Guhl/Koller/SChny der/Druey Das Schweizerische Obligationenrecht, 9. Aufl., 2000, S. 222. Der Rsp. zuneigend aber ENG E L Traité des obligations en droit suisse, 2. Aufl., 1997, S. $593 \mathrm{ff}$.

58 Schweizerisches Bundesgericht, Urteil vom 12.6.2008, Az. 4A_16/2008.

59 OGH in RdW 1984, 9; JBl 1986, 526. KOzIOL/Welser Bürgerliches Recht, Bd. II, 12. Aufl., 2001, S. 277 m. w. N. in Fn. 128.

60 SChlechtriem (Fn. 40), S. 646 Rdn. 476.

61 OGH JBl 1997, 37, 39 


\section{b) Frankreich}

Auch das französische Recht kennt keine den Konflikt regelnde Norm. Die Rückforderungssperre wird unmittelbar aus den römisch-rechtlichen Prinzipien »nemo auditur propriam turpitudinem allegans « und »in pariturpitudine melior est causa possidentis « hergeleitet. ${ }^{62}$ Rechtschutzverweigerungs- und Straftheorie werden eher kritisch als Entscheidungsgrundlagen diskutiert. ${ }^{63}$ Man bemüht sich, die Sittenwidrigkeit von der bloßen Verbotswidrigkeit abzugrenzen, beachtet den Normzweck als Auslegungskriterium, lässt sich aber auch - entgegen unserer Auffassung - auf einen Vergleich der Schwere der Verstöße der Parteien ein. ${ }^{64}$ Letzteres mein man aus dem Grundsatz »in pari turpitudine ..." ableiten zu können. Infolgedessen ist man von einem kohärenten und geschlossenen Regelungssystem sehr weit entfernt. ${ }^{65}$ Dies führt zwangsläufig zu einer großen Rechtsunsicherheit, den ein Teil der Lehre aber mit der Überlegung meint rechtfertigen zu können, es sei sinnvoll, dass die sich außerhalb der Rechtsordnung bewegenden Parteien den Ausgang des Prozesses nicht kalkulieren könnten, weil diese Unsicherheit eine Abschreckungswirkung entfalte. ${ }^{66} \mathrm{Zu}$ Recht hat Honsell diese Argumentation als "grotesk " zurückgewiesen, weil sie »in letzter Konsequenz zur Preisgabe aller rechtsstaatlichen Grundsätze im Bereich sittenwidriger Geschäfte « führt. ${ }^{67}$ Das französische Recht bietet also wenig Hilfreiches zu unserem Thema.

\section{c) England}

Dagegen sind die im Common Law entwickelten Grundsätze zur Rückabwicklung von gesetzes- oder sittenwidrigen Verträgen interessant. ${ }^{68}$ Schon 1775 wurde das non-recovery principle entwickelt, das besagt: »Let the estate lie, where it falls. « Die Grundsätze und die Begründung, die Lord Mansfield aufgestellt hat, zeigen die enge Verwandtschaft mit dem römisch-rechtlich geprägten kontinentaleuropäischen Rechtsdenken:

"The principle of public policy is this: ex dolo malo non oritur actio. No court will lend its aid to a man who founds his cause of action upon an immoral or an illegal act. If, from the plaintiff's own stating or otherwise, the cause of action appears to arise ex turpi causa, or the transgression of a positive law of this country, there the court says he has no right to be assisted. It is upon that ground the court goes; not for the sake of the defendant, but because they will not lend their aid to such a plaintiff. So if the plaintiff and defendant were to change sides, and the defendant was to bring his action against the plaintiff, the latter would then have the advantage of it; for where both are equally in fault, potior est conditio defendentis. ${ }^{69}$

Der letzte Satz (where both are equally in fault, potior est conditio defendentis) entspricht $\$ 817$ S. 2. In neuerer Zeit wird betont, es handele sich nicht um ein Prinzip der materiellen Gerechtigkeit, sondern lediglich um eine rechtspolitische Maxime, bei deren Anwendung notfalls auch Ungerechtigkeiten in Kauf genommen werden müssten.$^{70}$ Dies entspricht dem von uns geforderten Verbot, jenseits von Normzweck und Generalprävention Parteiinteressen abzuwägen. Dieser Gedanke ist auch in der englischen Literatur zur Begründung der Kondiktionssperre bei beiderseitigem Gesetzesverstoß bzw. beiderseitiger Sittenwidrigkeit anzutreffen: » The ... reason for the stringent rule is that the plaintiff must come to court with clean hands; moreover, it is not possible to distinguish between degrees of iniquity. «"11 Das Verbot nach Graden der Ungerechtigkeit zu unterscheiden ist nichts weiter als ein Verbot der Abwägung von Parteiinteressen. Der Vergleich mit dem englischen Recht unterstützt daher die von uns vertretene Auffassung. ${ }^{72}$

\section{Die Problematik des §817 S. 2 im Licht des Draft Common Frame of Reference}

Abschließend soll noch ein kurzer Blick auf die Entwicklung des europäischen Privatrechts geworfen werden. Im kürzlich von der
Study Group und der Acquis Group veröffentlichten Draft Common Frame of Reference ${ }^{73}$ findet sich im von der ungerechtfertigten Bereicherung handelnden 7. Buch im 6. Kapitel folgende Regelung (Art. VII - 6:103):

\section{Illegality}

Where a contract or other juridical act under which an enrichment is obtained is void or avoided because of an infringement of a fundamental principle (...) of mandatory rule of law, the enriched person is not liable to reverse the enrichment to the extent that the reversal would contravene the policy underlying the principle or rule.

Diese Regelung entspricht der hier vertretene Auffassung: Der Kondiktionsausschluss steht unter dem Vorbehalt der Verträglichkeit mit dem Zweck der Verbotsnorm, wozu auch generalpräventive Erwägungen zählen können. Darüber hinaus findet aber keine weitere Korrektur der Kondiktionssperre im Wege einer Abwägung von einzelfallbezogenen oder typisierten Parteiinteressen statt. Dies mag ein weiteres Argument für den BGH sein, die in der zweiten Schenkkreisentscheidung vorgenommene Kurskorrektur konsequent weiter zu verfolgen und damit die in der Schwarzarbeits- und Gewinnausschüttungsentscheidung vorgenommene Abwägung der Parteiinteressen in Zukunft zu unterlassen.

\section{Zusammenfassung}

Die zweite Schenkkreisentscheidung des BGH ist uneingeschränkt zu begrüßen. Sie stellt eine Korrektur der bisherigen Rechtsprechung zur Auslegung des $\$ 817$ S. 2 BGB dar. Die teleologische Reduktion des $\$ 817$ S. 2 BGB muss auf einer aus methodischer Sicht tragfähigen Grundlage erfolgen. Die Kondiktionssperre steht unter dem Vorbehalt, dass der Zweck der Verbotsnorm, insbesondere generalpräventive Erwägungen nicht die Zulassung der Kondiktion erzwingen. Wenn dies nicht der Fall ist, muss $\$ 817$ S. 2 BGB zur Anwendung kommen. Insbesondere darf keine Abwägung der Parteiinteressen - und zwar weder einzelfallbezogen noch typisierend - stattfinden. Insofern ist die Rechtsprechung an die gesetzgeberische Entscheidung des $\$ 817$ S. 2 BGB gebunden. Diese Forderung wird durch die Analyse der Hintergründe des $\$ 817$ S. 2 BGB und durch die rechtsvergleichende Untersuchung unterstützt. Insbesondere im Common Law wird eine Abwägung der Parteiinteressen abgelehnt. Die hier vertretene Ansicht wird zudem von Art. VII.-6:103 DCFR gestützt, der exakt die von uns vorgeschlagenen Kriterien normiert. Sie basiert auf einer Kombination der Lehren von der Generalprävention, vom Normzweckvorbehalt und von der Rechtsschutzverweigerung, die sich nicht gegenseitig ausschließen, sondern einander ergänzen. Es bleibt zu hoffen, dass der BGH in Zukunft den mit der zweiten Schenkkreisentscheidung eingeschlagenen Kurs konsequent weiterverfolgen wird.

62 Schlechtriem (Fn. 40), S. 646 Rdn. 477.

63 Guelfucci-Thibierge, Nullité, Nr. $903 \mathrm{ff}$.

64 SCHLEChtíiem (Fn. 40), S. 647 Rdn. 479

65 Schlechtriem (Fn. 40), S. 650 Rdn. 485.

66 Flour/Aubert I, 5. Aufl., Nr. 369.

67 HoNSELL (Fn. 27) S. 130.

68 Dazu SCHMidT-ReCla JZ 2008, S. 66.

69 Lord MAN F I E L D C. J. in Holmanv. Johnson, (1775) 1 Cowp. 341, 343. Zitiert nach Schlechtriem (Fn. 40), S. 228 Fn. 920.

70 Lord Goff of Chieveley in Tinsley v. Milligan, 1994, 1 A.C. 340 , 355.

71 Goff/Jones The Law of Restitution, 5, ed, 1998, S. 505.

72 Daran ändert auch die von SCH M I D T-RECLA JZ 2008, S. 66, erwähnte Tatsache, dass es vier Einschränkungen dieses Grundsprinzips (Schutz besonderer Personengruppe/Irrtum oder Drohung/non-reliance rule/timely withdrawal) gebe, nichts.

73 Dazu ausführlich ER N S T AcP 208 (2008), S. 248 ff. 